\title{
Current-induced torques in continuous antiferromagnetic textures
}

\author{
A. C. Swaving and R. A. Duine \\ Institute for Theoretical Physics, Utrecht University, Leuvenlaan 4, 3584 CE Utrecht, The Netherlands
}

(Received 10 December 2010; published 23 February 2011)

\begin{abstract}
We study the influence of an electric current on a continuous noncollinear antiferromagnetic texture. Despite the lack of a net magnetic moment, we find that the exchange interaction between conduction electrons and local magnetization generally results in current-induced torques that are nonzero and similar in phenomenology to spin-transfer torques in ferromagnets. We present the generalization of the nonlinear sigma model equation of motion for the Néel vector that includes these current-induced torques, and briefly discuss the resulting current-induced antiferromagnetic domain wall motion and spin-wave Doppler shift. We give an interpretation of our results using a unifying picture of current-induced torques in ferromagnets and antiferromagnets, in which they are viewed as being due to the current-induced spin polarization resulting from an effective spin-orbit coupling.
\end{abstract}

DOI: 10.1103/PhysRevB.83.054428

PACS number(s): 72.25.Ba, 75.30.Ds, 75.50.Pp

\section{INTRODUCTION}

Magnetoresistive phenomena in conducting ferromagnets, resulting from the interplay between spins of conduction electrons and the magnetization, are well known. They play a major role in new technologies and are key to the recently awarded Nobel prize for giant magnetoresistance. ${ }^{1,2}$ Recently, the effect of a spin current on magnetization dynamics (called spin transfer) has been investigated in detail. ${ }^{3-6}$ Although applications, mainly to memory storage technology, are an important driving factor behind this research, spin transfer is also physically interesting in its own right. It can be understood as follows.

Consider a conducting ferromagnet far below its critical temperature such that it is described by a unit vector $\boldsymbol{\Omega}(\boldsymbol{x}, t)$ in the direction of magnetization. Its dynamics in the presence of an effective field $\boldsymbol{H}_{\text {eff }}$ (in units of $s^{-1}$ ) is determined by

$$
\frac{\partial \boldsymbol{\Omega}}{\partial t}=\boldsymbol{\Omega} \times \boldsymbol{H}_{\mathrm{eff}}+\frac{J}{\hbar} \boldsymbol{\Omega} \times \nabla^{2} \boldsymbol{\Omega},
$$

where we have ignored magnetization relaxation, and the effective field contains (in the first instance) contributions from the anisotropy of the system and the external field. Furthermore, $J$ is the exchange constant favoring alignment of neighboring spins. In the presence of conduction electrons, the effective field contains an additional contribution due to the $s$ - $d$ exchange coupling of the magnetization with the spin density $s$ of the conduction electrons given by $-\Delta \int d \boldsymbol{x} \boldsymbol{s} \cdot \boldsymbol{\Omega} / a^{3}$, with $\Delta$ the exchange splitting and $a$ the lattice constant. (Although the $s$ - $d$ model is convenient to illustrate the physics involved, the conclusions drawn are qualitatively valid for the Stoner model as well.) In equilibrium, i.e., without a current, this spin density will be in the plane of the magnetization. However, when a current is applied, the spin density acquires a component $\left\langle\boldsymbol{s}_{\perp}\right\rangle$ perpendicular to the plane of magnetization. It is this component of the spin density that leads to current-driven dynamics of the magnetization ${ }^{7}$ by contributing to the effective field as

$$
\left.\boldsymbol{H}_{\text {eff }}\right|_{\text {current }}=\frac{\Delta a^{3}}{\hbar}\left\langle\boldsymbol{s}_{\perp}\right\rangle .
$$

To lowest order in the current and the gradient of the magnetization, and using an adiabatic assumption based on the fact that the electron dynamics is fast compared to the time variation of the magnetization, the out-of-plane spin density is

$$
\left\langle\boldsymbol{s}_{\perp}\right\rangle=\frac{\hbar}{\Delta a^{3}} \boldsymbol{\Omega} \times\left(\boldsymbol{v}_{s} \cdot \nabla\right) \boldsymbol{\Omega},
$$

where the velocity $\boldsymbol{v}_{s}$, defined via the above equation, is proportional to the electric current. Inserting the above contribution to $\boldsymbol{H}_{\text {eff }}$ in Eq. (1), we find that, in the steady-state transport situation, the contribution of the current-induced torques to the equation of motion for the magnetization direction is given by

$$
\left.\frac{\partial \boldsymbol{\Omega}(\boldsymbol{x}, t)}{\partial t}\right|_{\text {current }}=-\left(\boldsymbol{v}_{s} \cdot \nabla\right) \boldsymbol{\Omega}(\boldsymbol{x}, t) .
$$

The current-dependent velocity is, in the absence of spin-orbit coupling and spin-flip scattering, fixed by spin conservation as follows. Integrating Eq. (4) over the length of the ferromagnet in the direction $\hat{r}$ of the current leads to the total change in angular momentum $\int d r \partial \boldsymbol{\Omega}(\boldsymbol{x}, t) /\left.\partial t\right|_{\text {current }}=\boldsymbol{v}_{s} \cdot \hat{r}\left[\left.\boldsymbol{\Omega}\right|_{\text {in }}-\left.\boldsymbol{\Omega}\right|_{\text {out }}\right]$, where the current flows from in to out. By spin conservation, this change in angular momentum is proportional to the change in the spin current $\boldsymbol{J}_{s}^{\alpha}$ after it has passed through the ferromagnet, i.e., $\int d r \partial \Omega^{\alpha}(\boldsymbol{x}, t) /\left.\partial t\right|_{\text {current }} \propto \boldsymbol{J}_{s, \text { in }}^{\alpha}-\boldsymbol{J}_{s, \text { out }}^{\alpha}$. Using that $\boldsymbol{J}_{s}^{\alpha}=P \boldsymbol{J}_{c} \Omega^{\alpha}$, with $P$ the polarization of the charge current $\boldsymbol{J}_{c}$ in the ferromagnet, we find that $\boldsymbol{v}_{s} \propto P \boldsymbol{J}_{c}$. This argument shows that the current-induced torque in Eq. (4) results from transfer of angular momentum from conduction electrons to magnetization and is hence called a spin-transfer torque.

Well-known examples of dynamics resulting from these spin-transfer torques involve spin waves and domain walls. ${ }^{8-19}$ For spin waves, a Doppler shift in the dispersion relation is found once a current is applied. ${ }^{8,9}$ This shift is linearly proportional to $\boldsymbol{k}$, where the proportionality constant is given by $\boldsymbol{v}_{s}$ and the dispersion that follows from Eqs. (1)-(4) is then given by $\hbar \omega_{\boldsymbol{k}}=J \boldsymbol{k}^{2}+\hbar \boldsymbol{v}_{s} \cdot \boldsymbol{k}$, where we have ignored anisotropy and external fields. Recent experiments have successfully measured such current-induced spin-wave Doppler shifts. ${ }^{19}$ Current-induced domain wall motion $^{10-18}$ is understood as follows: In the absence of any 
pinning and damping, $\boldsymbol{\Omega}_{0}\left(\boldsymbol{x}-\boldsymbol{v}_{s} t\right)$ is a solution to Eq. (4), where $\boldsymbol{\Omega}_{0}(\boldsymbol{x})$ is a time-independent solution of Eq. (1), i.e., the equation without the effects of the applied current. For the case in which $\boldsymbol{\Omega}_{0}(\boldsymbol{x})$ corresponds to a domain wall texture, this means that the velocity of the domain wall is just $\boldsymbol{v}_{s}$, an estimate which turns out to be reasonable even when pinning and damping are present. ${ }^{10,11}$ These results are also understood by realizing that Eq. (4) shows that it is possible to get the equation of motion of $\boldsymbol{\Omega}$ with an applied current by substituting $\frac{\partial}{\partial t} \rightarrow \frac{\partial}{\partial t}+\boldsymbol{v}_{s} \cdot \nabla$ into the equation of motion without current.

In antiferromagnets, adjacent lattice sites have opposing magnetic moments and thus form two sublattices with opposite magnetization leaving no net magnetization. They are characterized by a Néel vector $\boldsymbol{n}_{\boldsymbol{j}}=(-1)^{j_{x}+j_{y}+j_{z}} \boldsymbol{\Omega}_{\boldsymbol{j}}$, where the vector $\boldsymbol{j}$ is a vector with components $\left(j_{x}, j_{y}, j_{z}\right)$ and labels the lattice sites $\boldsymbol{x}_{\boldsymbol{j}}=\boldsymbol{j} a$. In this paper, we show that, in the presence of an electric current in an antiferromagnetic conductor, there is in the continuum limit and to lowest order in the Néelvector gradient and the current an out-of-plane spin density given by

$$
\left\langle\boldsymbol{s}_{\perp}\right\rangle=\frac{\hbar}{\Delta a^{3}} \boldsymbol{n} \times(\boldsymbol{v} \cdot \nabla) \boldsymbol{n} .
$$

Here, the velocity $\boldsymbol{v}$, defined via the above equation, is proportional to the current and the antiferromagnetic equivalent of the velocity $\boldsymbol{v}_{s}$ that was introduced in describing current-induced torques in ferromagnets. Although the form of the spin density is the same as for a ferromagnet (albeit that magnetization direction $\boldsymbol{\Omega}$ is replaced by Néel vector $\boldsymbol{n}$ ), the velocity $\boldsymbol{v}$ can not be determined from macroscopic spin-conservation arguments, as in the case of the ferromagnet, but instead needs to be determined by a microscopic calculation. An example of such a calculation is presented in Sec. III. We show that $\boldsymbol{v}$ is generally not zero and can, in principle, be of the same order of magnitude as $\boldsymbol{v}_{s}$, which is of the order of $1-100 \mathrm{~m} / \mathrm{s}$ for a typical ferromagnetic alloy such as permalloy, by calculating $\boldsymbol{v}_{s}$ and $\boldsymbol{v}$ for a toy-model ferromagnetic and antiferromagnetic metal. In Sec. IV, we explain our results using a unifying picture of current-induced torques in ferromagnetic and antiferromagnetic textures that is built on the notion of effective spin-orbit coupling induced by a noncollinear magnetic texture, and we end with a discussion and conclusion in Sec. V.

Previous work on current-induced torques in antiferromagnetic metals, considered mainly single-domain layered structures, ${ }^{20-25}$ and the situation of an antiferromagnetic domain wall was considered from an $a b$ initio point of view. ${ }^{26}$ Here, the general phenomenology of current-induced torques in spatially smooth and slowly varying antiferromagnetic textures is presented. To make the paper more self-contained, we start by deriving the equation of motion for the Néel vector in a position- and time-dependent external field, generalizing the results from Ref. 27. From this equation of motion, the generalization of the nonlinear sigma model equation of motion to the current-carrying situation follows via Eqs. (2) and (5). The consequences of this equation of motion for current-induced domain wall motion and the spin-wave spectrum are briefly discussed.

\section{NONLINEAR SIGMA MODEL EQUATION OF MOTION WITH CURRENT-INDUCED TORQUES}

We consider a system of classical spins on lattice sites $x_{j}=j a$, with $a$ the lattice constant. The direction of each spin is indicated by the unit vector $\boldsymbol{\Omega}_{j}$. Its equation of motion, ignoring damping and anisotropy, is given by

$$
\frac{\partial \boldsymbol{\Omega}_{j}}{\partial t}=-\frac{J}{\hbar} \boldsymbol{\Omega}_{j} \times\left(\boldsymbol{\Omega}_{j-1}+\boldsymbol{\Omega}_{j+1}\right)+\boldsymbol{\Omega}_{j} \times \boldsymbol{H}_{\mathrm{eff}},
$$

with $J$ the antiferromagnetic exchange coupling and $\boldsymbol{H}_{\text {eff }}$ a position- and time-dependent effective field in which we have absorbed dimensionful prefactors. Note that we consider a onedimensional lattice. The three-dimensional generalization will be presented elsewhere. To incorporate the antiferromagnetic alignment of the spins and to enable a gradient expansion, we introduce

$$
\begin{gathered}
\boldsymbol{A}_{m}=\boldsymbol{\Omega}_{j}, \\
\boldsymbol{B}_{m}=\boldsymbol{\Omega}_{j+1},
\end{gathered}
$$

which obey the equation of motion

$$
\begin{aligned}
& \frac{\partial \boldsymbol{A}_{m}}{\partial t}=-\frac{J}{\hbar} \boldsymbol{A}_{m} \times\left(\boldsymbol{B}_{m-1}+\boldsymbol{B}_{m}\right)+\boldsymbol{A}_{m} \times \boldsymbol{H}_{\mathrm{eff}}, \\
& \frac{\partial \boldsymbol{B}_{m}}{\partial t}=-\frac{\boldsymbol{J}}{\hbar} \boldsymbol{B}_{m} \times\left(\boldsymbol{A}_{m}+\boldsymbol{A}_{m+1}\right)+\boldsymbol{B}_{m} \times \boldsymbol{H}_{\mathrm{eff}},
\end{aligned}
$$

and where the index $m$ labels pairs of adjacent sites $x_{j}$ of the lattice. The next step is to introduce the magnetization

$$
\boldsymbol{M}_{m}=\frac{\boldsymbol{A}_{m}+\boldsymbol{B}_{m}}{2}
$$

and the Néel vector

$$
\boldsymbol{n}_{m}=\frac{\boldsymbol{A}_{m}-\boldsymbol{B}_{m}}{2},
$$

which are orthogonal to each other and obey $\boldsymbol{M}_{m}^{2}+\boldsymbol{n}_{m}^{2}=1$.

We now proceed as follows: First, we derive equations of motion for $\boldsymbol{n}_{m}$ and $\boldsymbol{M}_{m}$ by eliminating $\boldsymbol{A}_{m}$ and $\boldsymbol{B}_{m}$ from Eq. (8) in favor of the magnetization and the Néel vector. Second, we take the continuum limit and introduce derivatives via $f_{m \pm 1} \simeq$ $f_{m} \pm 2 a \partial f / \partial x+2 a^{2} \partial^{2} f / \partial x^{2}$. We expand up to second order in derivatives and work to first order in the effective field $\boldsymbol{H}_{\text {eff }}$. With respect to this, it is important to note that, as we will show [Eq. (12)], $\boldsymbol{M}$ is first order in $\partial \boldsymbol{n} / \partial x, \partial \boldsymbol{n} / \partial t$, and $\boldsymbol{H}_{\text {eff }}$. Furthermore, $\boldsymbol{H}_{\text {eff }}$ is also first order in $\partial \boldsymbol{n} / \partial x$ via Eq. (5). The final result for the coupled equations of motion for the Néel vector and the magnetization is ${ }^{27}$

$$
\begin{gathered}
\frac{\partial \boldsymbol{n}}{\partial t}=-\frac{2 J}{\hbar}\left(2 \boldsymbol{n} \times \boldsymbol{M}+a \boldsymbol{n} \times \frac{\partial \boldsymbol{n}}{\partial x}\right)+\boldsymbol{n} \times \boldsymbol{H}_{\mathrm{eff}}, \\
\frac{\partial \boldsymbol{M}}{\partial t}=-\frac{2 J a}{\hbar}\left[\frac{\partial}{\partial x}(\boldsymbol{M} \times \boldsymbol{n})-\boldsymbol{n} \times \frac{\partial^{2} \boldsymbol{n}}{\partial x^{2}}\right]+\boldsymbol{M} \times \boldsymbol{H}_{\mathrm{eff}} .
\end{gathered}
$$

The first of these two equations is rewritten by taking the cross product with $\boldsymbol{n}$ to yield

$$
\boldsymbol{M}=\frac{\hbar}{4 J} \boldsymbol{n} \times \frac{\partial \boldsymbol{n}}{\partial t}-\frac{a}{2} \frac{\partial \boldsymbol{n}}{\partial x}+\frac{\hbar}{4 J} \boldsymbol{H}_{\mathrm{eff}} .
$$

Here we assume that the effective field is perpendicular to the Neel vector. 
This is inserted in the second equation in Eq. (11) and gives

$$
\begin{aligned}
\boldsymbol{n} \times & \left(\frac{\partial^{2} \boldsymbol{n}}{\partial t^{2}}-c^{2} \frac{\partial^{2} \boldsymbol{n}}{\partial x^{2}}\right)+\frac{\partial \boldsymbol{H}_{\mathrm{eff}}}{\partial t} \\
& =\left(\boldsymbol{n} \times \frac{\partial \boldsymbol{n}}{\partial t}\right) \times \boldsymbol{H}_{\mathrm{eff}}-\frac{2 J a}{\hbar}\left(\frac{\partial \boldsymbol{H}_{\mathrm{eff}}}{\partial x}\right) \times \boldsymbol{n}
\end{aligned}
$$

where we have introduced the spin-wave velocity $c=2 \mathrm{Ja} / \hbar$. Inserting the result for the current-induced effective field from Eq. (5), to be proven in the next section, we arrive at the generalization of the nonlinear sigma model equation of motion that includes current-induced torques in antiferromagnetic metals:

$$
\begin{array}{r}
\boldsymbol{n} \times\left(\frac{\partial^{2} \boldsymbol{n}}{\partial t^{2}}-c^{2} \frac{\partial^{2} \boldsymbol{n}}{\partial x^{2}}\right)+\frac{\partial}{\partial t}\left[\boldsymbol{n} \times v \frac{\partial \boldsymbol{n}}{\partial x}\right] \\
=-\left\{\boldsymbol{n} \cdot\left[\frac{\partial \boldsymbol{n}}{\partial t} \times v \frac{\partial \boldsymbol{n}}{\partial x}\right]\right\} \boldsymbol{n}-c v \frac{\partial^{2} \boldsymbol{n}}{\partial x^{2}}
\end{array}
$$

where we mention again that the velocity $v$ is proportional to the transport current.

The antiferromagnetic spin-wave dispersion resulting from this equation is, again ignoring anisotropy and external fields, given by

$$
\omega_{k}=c k \sqrt{1 \mp i v / c+(v / 2 c)^{2}} \pm \frac{v k}{2} \simeq c k \pm \frac{v k}{2}
$$

to linear order in current for $v \ll c$. This shows that the dispersion has a similar Doppler shift to the ferromagnetic case. ${ }^{8,9}$ The difference with the ferromagnetic case is in the factor $1 / 2$ in the Doppler shift, resulting from the fact that the equation for $\boldsymbol{n}(x, t)$ is second order in time derivatives. With the definition in Eq. (5), the velocity $v$ is equal to the velocity of current-driven domain walls in the absence of damping, anisotropy, and pinning, as we discuss now. The corrections of $\mathcal{O}(v / c)$ under the square root in Eq. (15), which are unimportant for $v \ll c$ (which we assume is the typical situation), come from the last term in Eq. (14). Upon ignoring this term, the equation of motion in Eq. (14) allows for comoving solutions $n_{0}(\boldsymbol{x}-\boldsymbol{v} t$ ) (to first order in $\boldsymbol{v}$ ), from which we conclude that antiferromagnetic domain walls ${ }^{28}$ move with velocity $v$ proportional to the current in the absence of pinning and damping. Further justification of this estimate of the current-induced domain wall velocity is that the term that is neglected in this estimate is quadratic in spatial derivatives and, by itself, therefore, does not lead to domain wall motion, but only to modification of the spin-wave dispersion if $v$ becomes comparable to $c$.

In the next section, we will prove the form of the spin density in Eq. (5) that was assumed so far, and calculate $v$ for a simple model.

\section{TOY-MODEL ANTIFERROMAGNETIC METAL}

To confirm the conjecture $\left\langle\boldsymbol{s}_{\perp}\right\rangle \propto \boldsymbol{n} \times(\boldsymbol{v} \cdot \boldsymbol{\nabla}) \boldsymbol{n}$ and determine the current-dependent velocity $\boldsymbol{v}$, we consider a toy model of a conducting antiferromagnet within the Green's function formulation of Landauer-Büttiker transport theory. ${ }^{29}$ We use

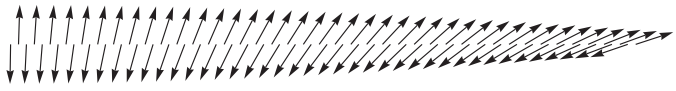

FIG. 1. Illustration of a smooth antiferromagnetic magnetization texture.

a tight-binding Hamiltonian given by $H=H_{S}+H_{L}+H_{I}$, where

$$
H_{S}=-t \sum_{\left\langle j, j^{\prime}\right\rangle ; \sigma} \psi_{j, \sigma}^{\dagger} \psi_{j^{\prime}, \sigma}-\sum_{j ; \sigma, \sigma^{\prime}} \psi_{j, \sigma}^{\dagger}\left[\frac{\Delta}{2} \boldsymbol{\Omega}_{j} \cdot \boldsymbol{\tau}_{\sigma, \sigma^{\prime}}\right] \psi_{j, \sigma^{\prime}}
$$

is the system Hamiltonian with $t$ the nearest-neighbor hopping amplitude and $\Delta$ the exchange energy, and $\psi_{j, \sigma}$ and $\psi_{j, \sigma}^{\dagger}$ are the electron annihilation and creation operators. The Hamiltonians for the leads $H_{L}$ and for the coupling between the leads and the system $H_{I}$, respectively, are similar but with $\Delta=$ 0 . To realize a transport current $I$, these leads have a chemical potential difference of $e|V|$. The magnetization texture is set to $\boldsymbol{\Omega}_{\boldsymbol{j}}=\left[(\mp 1)^{j} \sin \left(\frac{2 \pi a j}{\lambda}\right), 0,(\mp 1)^{j} \cos \left(\frac{2 \pi a j}{\lambda}\right)\right] \equiv(\mp 1)^{j} \boldsymbol{n}_{j}$, where $\lambda$ is the wavelength of the magnetic texture and the upper (lower) signs describe the antiferromagnetic (ferromagnetic) case. See Fig. 1 for an illustration of the antiferromagnetic texture.

In Fig. 2, we show the transmission probability as a function of $\epsilon_{F} / t$ for two different values of $\Delta / t$ and a lattice of 82 sites. The result for the ferromagnetic case is shown for comparison. The results are understood by noting that, for the collinear situation, the dispersion of the electrons in the antiferromagnet is

$$
\epsilon_{k, \pm}= \pm \sqrt{\Delta^{2}+4 t^{2} \cos ^{2}(k a)}
$$

where the + and - distinguish the two bands. The above dispersion has a gap between $-\sqrt{\Delta^{2}-4 t^{2}}$ and $\sqrt{\Delta^{2}-4 t^{2}}$, which shows up as a suppression in the transmission probability if the Fermi energy of the leads is put in this gap. For values outside the gap, the transmission probability approaches approximately 2 , corresponding to perfect transmission of the

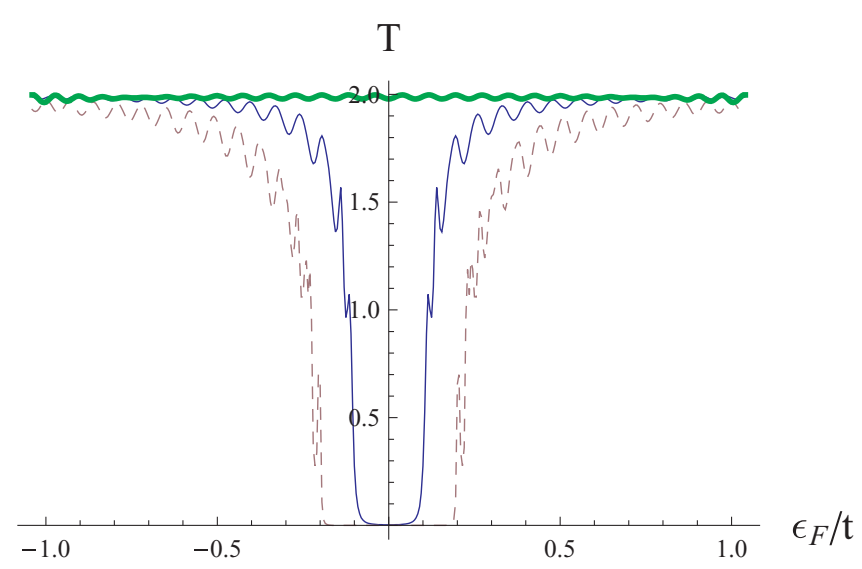

FIG. 2. (Color online) Transmission probability as a function of Fermi energy $\epsilon_{F} / t$ for $\Delta / t=0.1$ (solid lines) and $\Delta / t=0.2$ (dashed lines) for both the antiferromagnetic (thin lines) and ferromagnetic (thick line, only the $\Delta=0.2$ is shown and the $\Delta=0.1$ is similar) cases. We considered a lattice with 82 sites. 


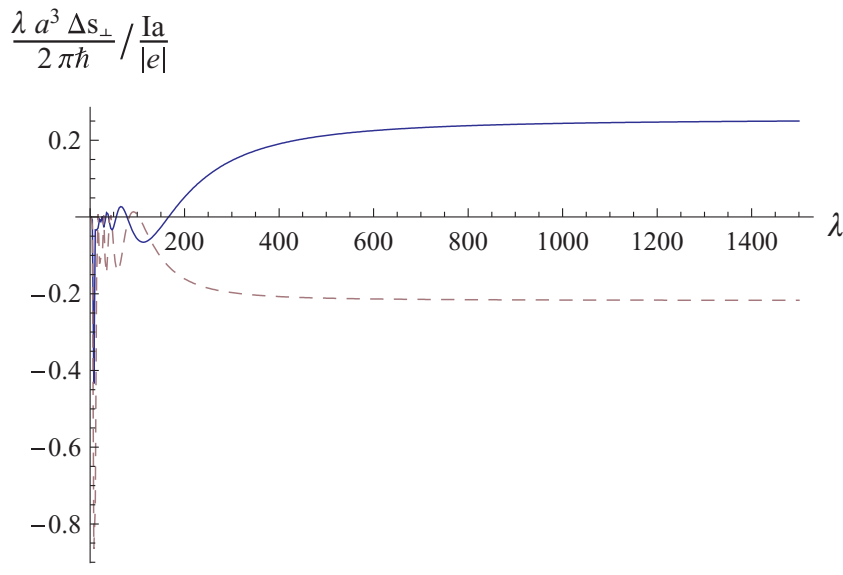

FIG. 3. (Color online) Plot of the current-induced out-of-plane spin density vs the antiferromagnetic texture wavelength $\lambda$. The solid line corresponds to $\Delta / t=0.1$ and the dashed line to $\Delta / t=0.2$. The Fermi energy $\epsilon_{F} / t=0.79$.

two spin states of the electron. The oscillations as a function of $\epsilon_{F}$ are due to finite-size effects. We have checked numerically that decreasing the size of the lattice decreases the period to these oscillations proportionally.

Next, we consider the spin density of the electrons. The magnetization texture is in the $x-z$ plane and in equilibrium, without current, the system only has nonzero spin densities $\langle\boldsymbol{s}\rangle=\left\langle\psi_{\sigma}^{\dagger} \boldsymbol{\tau}_{\sigma, \sigma^{\prime}} \psi_{\sigma^{\prime}}\right\rangle$ in this plane. For nonzero voltage, we numerically find a spin density $\left\langle\boldsymbol{s}_{\perp}\right\rangle$ in the $y$ direction that is constant in position, in agreement with Eq. (5). If Eq. (5) holds, then the velocity

$$
v \equiv \frac{a^{3} \Delta\left\langle s_{\perp}\right\rangle}{\hbar\left[\boldsymbol{n}_{j} \times \frac{\partial}{\partial x_{j}} \boldsymbol{n}_{j}\right]_{y}}=\frac{\lambda a^{3} \Delta\left\langle s_{\perp}\right\rangle}{2 \pi \hbar}
$$

should be independent of $\lambda$ in the long-wavelength limit. The numerical results shown in Fig. 3 confirm this. In this figure, the current-induced out-of-plane spin density, normalized to the current, is shown as a function of the wavelength of the antiferromagnetic texture. For increasing texture wavelength, the velocity approaches a constant value. The oscillations for small $\lambda$ are again due to finite-size effects, as we have found that they change period upon changing the system size. For comparison, we show in Fig. 4 the current-induced out-of-plane spin density versus magnetic-texture wavelength for a ferromagnet. Note that the long-wavelength limit is approached on a similar length scale as in the antiferromagnetic case. Although these results are obtained within a simple model, we expect that they are qualitatively valid in general. For example, we expect that, in the presence of disorder leading to a mean-free path $\ell$, the results are attenuated and the long-wavelength limit is approached as the magnetic-texture wavelength becomes much larger than the mean-free path (provided that the exchange coupling between the conduction electrons and magnetization is large).

Now that we have established that $\boldsymbol{v}$ approaches a constant in the long-wavelength limit, we take its long-wavelength limiting value as its definition and study its dependence on $\Delta$ and $\epsilon_{F}$. The result is shown in Fig. 5. Here, we show $v$ as a function of $\epsilon_{F}$ for two values of the exchange constant $\Delta$. It is

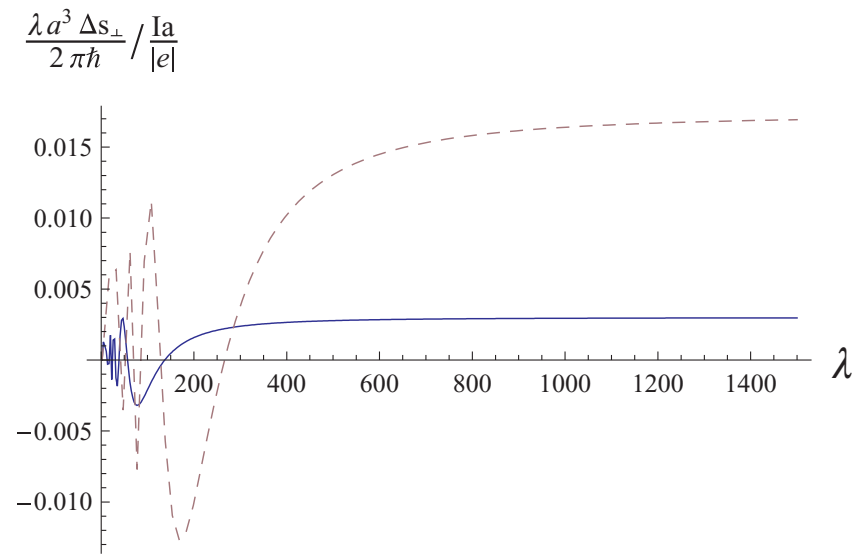

FIG. 4. (Color online) Plot of the current-induced out-of-plane spin density vs the texture wavelength $\lambda$ for a ferromagnet. The solid line corresponds to $\Delta / t=0.1$ and the dashed line to $\Delta / t=0.2$. The Fermi energy $\epsilon_{F} / t=0.79$.

clear from this figure that $\boldsymbol{v}$ depends strongly on the value of the exchange constant and the Fermi energy, at least within the model we study here. For values of the Fermi energy in the gap, the velocity becomes large because the current becomes small. In this regime, the model does not describe an antiferromagnetic metal and this regime is therefore not of interest to us. Note that the velocity is odd as a function of $\epsilon_{F}$, a result of the particle-hole symmetry of the band structure around zero energy. As in the results for the transmission probability in Fig. 2, the oscillations are due to finite-size effects.

For comparison, we show the results corresponding to the ferromagnetic case in Fig. 6. Note that there are similar oscillations as in the antiferromagnetic results, also due to finite-size effects. As discussed in the Introduction, the velocity $v_{s}$ is proportional to the charge current and its spin polarization. The charge current is approximately independent of $\epsilon_{F} / t$ because the transmission does not strongly depend on this parameter in the ferromagnetic case (compare Fig. 2). The oscillations in Fig. 6 are, therefore, primarily due to oscillations

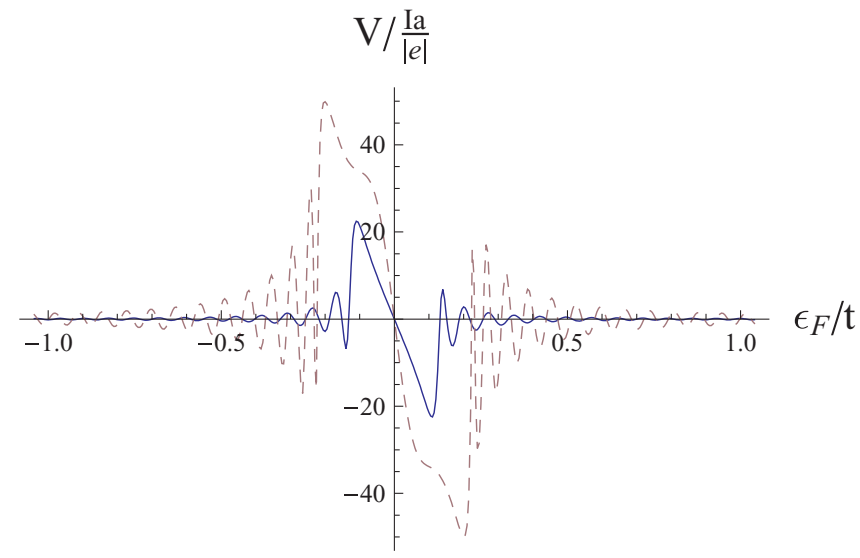

FIG. 5. (Color online) Plot of the velocity $v$ that parametrizes current-induced torques in bulk antiferromagnets vs $\epsilon_{F} / t$. The solid line corresponds to $\Delta / t=0.1$ and the dashed line to $\Delta / t=0.2$. The number of lattice sites is equal to 82 . 


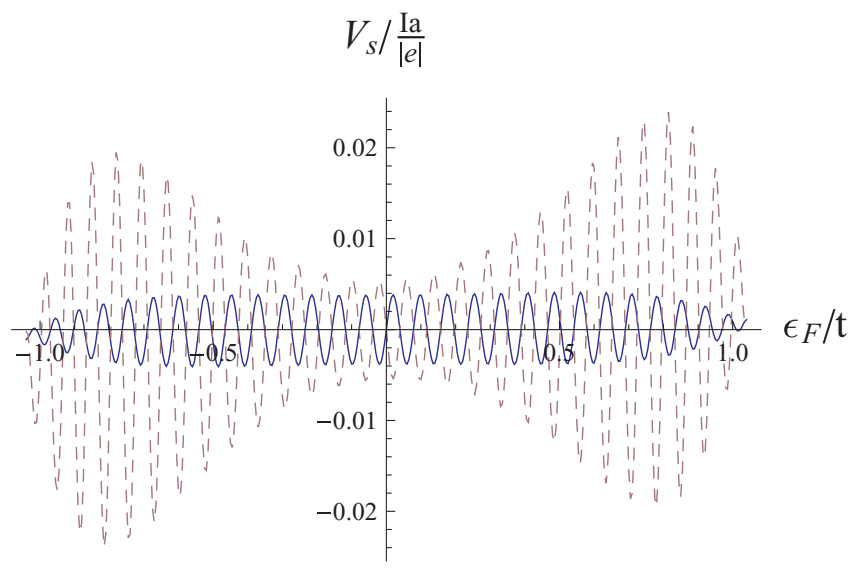

FIG. 6. (Color online) Plot of the velocity $\boldsymbol{v}_{s}$ that parametrizes current-induced torques in bulk ferromagnets vs $\epsilon_{F} / t$. The solid line corresponds to $\Delta / t=0.1$ and the dashed line to $\Delta / t=0.2$. The number of lattice sites is taken to be 82 .

in the spin polarization of the charge current. We have checked this numerically. The slight asymmetry around zero Fermi energy is due to the finite bias voltage between the left and right leads.

Although we consider a specific simple model here, the fact that our numerical results show that the results for the ferromagnetic and antiferromagnetic cases are of similar magnitude indicates that the velocities $\boldsymbol{v}_{s}$ and $\boldsymbol{v}$, respectively, parametrizing current-induced torques in ferromagnets and antiferromagnets, can in principle be of the same order of magnitude.

\section{EFFECTIVE SPIN-ORBIT COUPLING}

This numerical analysis confirms that, when a current is applied to an antiferromagnetic metal, there is a nonzero out-ofplane spin density that drives current-induced magnetization dynamics. We now give an interpretation for this spin density in terms of a current-induced spin polarization resulting from an effective spin-orbit coupling. We start with a system of electrons (mass $m$ ) moving in an antiferromagnetic texture $\boldsymbol{n}\left(\boldsymbol{x}_{j}\right)$ and scalar potential $V\left(\boldsymbol{x}_{j}\right)$, described by the Hamiltonian

$$
\mathcal{H}=\frac{p^{2}}{2 m}+V\left(\boldsymbol{x}_{\boldsymbol{j}}\right)-\frac{\Delta}{2}(-1)^{j} \boldsymbol{n}\left(\boldsymbol{x}_{\boldsymbol{j}}\right) \cdot \boldsymbol{\tau},
$$

with $\boldsymbol{x}_{j}=j a$ the position of the $j$ th lattice site. We align the spin quantization axis of the conduction electrons to the local Néel vector by applying a SU(2) transformation $|\psi\rangle \rightarrow R|\psi\rangle$ to the wave function with $R^{-1} \boldsymbol{n} \cdot \boldsymbol{\tau} R=\tau^{z}$, which therefore diagonalizes the spin part of the Hamiltonian and gives the effective Hamiltonian

$$
\mathcal{H}_{\text {eff }}=\mathcal{H}_{0}-2 i J_{s, \alpha}^{\mu} a_{\alpha}^{\mu}(\boldsymbol{x})
$$

to first order in the gradient of $\boldsymbol{n}$, where $\mathcal{H}_{0}$ is the Hamiltonian in Eq. (19) with $\boldsymbol{n}=\hat{z}$. Here, $J_{s, \alpha}^{\mu}=\frac{\hbar}{2} \tau^{\mu} v_{0, \alpha}$ is the spin current with the velocity $v_{0, \alpha}=\frac{1}{\hbar} \frac{\partial \epsilon_{k}}{\partial k_{\alpha}}$ with $\epsilon_{\boldsymbol{k}}$ the dispersion corresponding to $\mathcal{H}_{0}$ and the gauge fields $a_{\alpha}^{\mu}(\boldsymbol{x}) \cong i \boldsymbol{n} \times\left.\nabla_{\alpha} \boldsymbol{n}\right|_{\mu} \cdot{ }^{30}$ Substituting the latter into Eq. (20) leads to the effective Hamiltonian

$$
\begin{aligned}
\mathcal{H}_{\text {eff }} & =\mathcal{H}_{0}+\left(\boldsymbol{n} \times\left[\frac{\partial \epsilon_{\boldsymbol{k}}}{\partial k_{\alpha}} \nabla_{\alpha}\right] \boldsymbol{n}\right) \cdot \boldsymbol{\tau} \\
& \equiv \mathcal{H}_{0}-\boldsymbol{B}_{\mathrm{eff}} \cdot \boldsymbol{\tau},
\end{aligned}
$$

where $\boldsymbol{B}_{\text {eff }}$ is a momentum-dependent fictitious magnetic field that can be viewed as an effective spin-orbit coupling resulting from the noncollinear antiferromagnetic texture. (Note that this effective spin-orbit coupling is different from the result of Ref. 31 for a collinear antiferromagnet.) Since the effective magnetic field is linearly proportional to the velocity operator, when an electric field is applied, $\boldsymbol{B}_{\text {eff }}$ is nonzero. The resulting Zeeman splitting of the electron spins due to $\boldsymbol{B}_{\text {eff }}$ results in a current-induced spin polarization that is aligned with the effective magnetic field and, since $\boldsymbol{B}_{\text {eff }} \propto \boldsymbol{n} \times \nabla \boldsymbol{n}$, consequently, is perpendicular to both the magnetization and its gradient. It is this current-induced spin polarization that contributes to the effective field for the magnetization and leads to current-induced torques. The above argument holds in an analogous form for ferromagnets and confirms that the magnetization-direction dependence of $\left\langle\boldsymbol{s}_{\perp}\right\rangle$ for ferromagnets is the same as its Néel vector dependence for antiferromagnets. Note that, at the level of the effective Hamiltonian in Eq. (21), the main difference between the antiferromagnetic and ferromagnetic cases is the difference in $\mathcal{H}_{0}$, which in the ferromagnetic case contains a constant exchange splitting and in the antiferromagnetic case an alternating one. Current-induced spin polarization has been studied in paramagnetic semiconductors, ${ }^{32-34}$ which show that it does not require a net nonzero exchange splitting, which, in turn, explains why the current-induced torques in antiferromagnets are generally nonzero.

\section{DISCUSSION AND CONCLUSIONS}

In this paper, we have demonstrated that, in an antiferromagnetic metal, a steady-state transport current generates a current-induced out-of-plane spin density, resulting in torques on the magnetization. This spin density is parametrized by a velocity $\boldsymbol{v}$ that is proportional to the current. We have presented the generalization of the nonlinear sigma model equation of motion for antiferromagnetic magnetization dynamics in an antiferromagnetic metal in the presence of a transport current. From this equation of motion, we have found a current-induced shift of the spin-wave dispersion and also that the current-induced torques lead to current-driven antiferromagnetic domain wall motion.

One of our findings is that the form of the current-induced spin density, expressed in terms of the Néel vector, is similar in form to the current-induced spin density in ferromagnets with the Néel vector replaced by the magnetization direction. We have ignored conduction-electron spin relaxation, which, in the ferromagnetic case, is known to result in an additional contribution $-\beta \nabla \Omega$ to the spin density that is parametrized by the dimensionless constant $\beta .^{35,36}$ In future work, we intend to investigate if similar corrections are present in the antiferromagnetic situation as well. Moreover, we intend to explore the consequences of this equation of motion for the current-driven motion of antiferromagnetic 
domain walls, also taking into account anisotropy and magnetization relaxation. Further studies will also include exploring other aspects of the effective spin-orbit coupling discussed in this paper.

Note added in proof. In a recent preprint (Ref. 37) Hals, Tserkovnyak, and Brataas, investigate the effects of relaxation on current-induced dynamics in antiferromagnets.

\section{ACKNOWLEDGMENTS}

This work was supported by the Stichting voor Fundamenteel Onderzoek der Materie (FOM), the Netherlands Organization for Scientific Research (NWO), and the European Research Council (ERC) under the Seventh Framework Program (FP7).
${ }^{1}$ P. Grünberg, R. Schreiber, Y. Pang, M. B. Brodsky, and H. Sowers, Phys. Rev. Lett. 57, 2442 (1986).

${ }^{2}$ M. N. Baibich, J. M. Broto, A. Fert, F. Nguyen Van Dau, F. Petroff, P. Etienne, G. Creuzet, A. Friederich, and J. Chazelas, Phys. Rev. Lett. 61, 2472 (1988).

${ }^{3}$ J. C. Slonczewski, J. Magn. Magn. Mater. 159, L1 (1996).

${ }^{4}$ L. Berger, Phys. Rev. B 54, 9353 (1996).

${ }^{5}$ M. Tsoi, A. G. M. Jansen, J. Bass, W.-C. Chiang, M. Seck, V. Tsoi, and P. Wyder, Phys. Rev. Lett. 80, 4281 (1998).

${ }^{6}$ E. B. Myers, D. C. Ralph, J. A. Katine, R. N. Louie, and R. A. Buhrman, Science 285, 867 (1999).

${ }^{7}$ A. S. Núñez and A. H. MacDonald, Solid State Commun. 139, 31 (2006).

${ }^{8}$ Ya. B. Bazaliy, B. A. Jones, and Shou-Cheng Zhang, Phys. Rev. B 57, R3213 (1998).

${ }^{9}$ J. Fernández-Rossier, M. Braun, A. S. Núñez, and A. H. MacDonald, Phys. Rev. B 69, 174412 (2004).

${ }^{10}$ G. Tatara and H. Kohno, Phys. Rev. Lett. 92, 086601 (2004); 96, 189702 (2006).

${ }^{11}$ A. Thiaville, Y. Nakatani, J. Miltat, and Y. Suzuki, Europhys. Lett. 69, 990 (2005).

${ }^{12}$ J. Grollier, P. Boulenc, V. Cros, A. Hamzi, A. Vaurs, A. Fert, and G. Faini, Appl. Phys. Lett. 83, 509 (2003).

${ }^{13}$ M. Tsoi, R. E. Fontana, and S. S. P. Parkin, Appl. Phys. Lett. 83, 2617 (2003).

${ }^{14}$ A. Yamaguchi, T. Ono, S. Nasu, K. Miyake, K. Mibu, and T. Shinjo, Phys. Rev. Lett. 92, 077205 (2004).

${ }^{15}$ M. Kläui, C. A. F. Vaz, J. A. C. Bland, W. Wernsdorfer, G. Faini, E. Cambril, L. J. Heyderman, F. Nolting, and U. Rüdiger, Phys. Rev. Lett. 94, 106601 (2005).

${ }^{16}$ G. S. D. Beach, C. Knutson, C. Nistor, M. Tsoi, and J. L. Erskine, Phys. Rev. Lett. 97, 057203 (2006).
${ }^{17}$ M. Hayashi, L. Thomas, C. Rettner, R. Moriya, and S. S. P. Parkin, Nat. Phys. 3, 21 (2007).

${ }^{18}$ M. Yamanouchi, D. Chiba, F. Matsukura, and H. Ohno, Nature (London) 428, 539 (2004).

${ }^{19}$ V. Vlaminck and M. Bailleul, Science 322, 410 (2008).

${ }^{20}$ A. S. Núñez, R. A. Duine, P. M. Haney, and A. H. MacDonald, Phys. Rev. B 73, 214426 (2006).

${ }^{21}$ Z. Wei, A. Sharma, A. S. Nunez, P. M. Haney, R. A. Duine, J. Bass, A. H. MacDonald, and M. Tsoi, Phys. Rev. Lett. 98, 116603 (2007).

${ }^{22}$ S. Urazhdin and N. Anthony, Phys. Rev. Lett. 99, 046602 (2007).

${ }^{23}$ X. L. Tang, H. W. Zhang, H. Su, Z. Y. Zhong, and Y. L. Jing, Appl. Phys. Lett. 91, 122504 (2007).

${ }^{24}$ D. Herranz, R. Guerrero, R. Villar, F. G. Aliev, A. C. Swaving, R. A. Duine, C. van Haesendonck, and I. Vavra, Phys. Rev. B 79, 134423 (2009).

${ }^{25}$ H. Gomonay and V. Loktev, J. Magn. Soc. Jpn. 32, 535 (2008).

${ }^{26}$ Y. Xu, S. Wang, and K. Xia, Phys. Rev. Lett. 100, 226602 (2008).

${ }^{27}$ H.-J. Mikeska and M. Steiner, Adv. Phys. 40, 191 (1991).

${ }^{28}$ N. Papanicolaou, Phys. Rev. B 51, 15062 (1995).

${ }^{29}$ S. Datta, Electronic Transport in Mesoscopic Systems (Cambridge University Press, 1995).

${ }^{30}$ B. I. Shraiman and E. D. Siggia, Phys. Rev. Lett. 61, 467 (1988).

${ }^{31}$ R. Ramazashvili, Phys. Rev. Lett. 101, 137202 (2008).

${ }^{32}$ V. Sih, R. C. Myers, Y. K. Kato, W. H. Lau, A. C. Gossard, and D. D. Awschalom, Nat. Phys. 1, 31 (2005).

${ }^{33}$ V. M. Edelstein, Solid State Commun. 73, 233 (1990).

${ }^{34}$ A. G. Aronov and Yu. B. Lyanda Geller, Pis'ma Zh. Eksp. Teor. Fiz. 50, 398 (1989) [JETP Lett. 50, 431 (1989)].

${ }^{35}$ S. Zhang and Z. Li, Phys. Rev. Lett. 93, 127204 (2004).

${ }^{36}$ S. E. Barnes and S. Maekawa, Phys. Rev. Lett. 95, 107204 (2005).

${ }^{37}$ K. M. D. Hals, Y. Tserkovnyak, and A. Brataas, e-print arXiv:1012.5655v1 [cond-mat.mes-hall]. 